\title{
Directionality Effects via Distance-based Penalty Scaling
}

\author{
Sharon Inkelas and Eric Wilbanks \\ University of California, Berkeley
}

\section{Introduction}

In this paper we develop a new proposal for distance-based penalty scaling in Optimality-theoretic analyses, including Harmonic Grammar. We apply this technique to the analysis of two phonological phenomena, both of which have posed challenges to implementation using standard constraint-based methods: directionality effects and bounded domain windows. We show that distance-based penalty scaling handles both effects in a straightforward and unified manner.

Directionality effects, in particular, are widely acknowledged as challenging for surface-oriented constraint-based models (see e.g. Zoll (1997); Hansson (2001); McCarthy (2003); Bakovic \& Rose (2014), among many others). Cases covered by our analysis include both unidirectional harmonies and stress assignment patterns, as well as more complex cases of 'opposite-edge' effects, in which two competing directionality effects co-exist. We show that the same scaling functions which offer a solution to directionality effects can be readily applied to model window effects (e.g., Kager (2012)), in which phonotactic constraints apply in only one portion of a domain.

\section{Proposal}

Distance-based penalty scaling of constraints involves increasing the violation penalty for a constraint by an amount based on the distance between the locus of violation and some designated boundary (on a projection/tier). This intuition is familiar inasmuch as it underpins the family of gradient Align constraints (McCarthy \& Prince (1993)). The novelty in our proposal lies, in part, in the application of distancebased scaling to Faithfulness and Markedness constraints. As we discuss towards the end of this paper, enhancing Faithfulness and Markedness constraints with distance-based scaling raises the question of whether Alignment constraints, per se, are still needed.

2.1 Toy example: anticipatory sibilant harmony To illustrate distance-based penalty scaling, we consider a toy example of anticipatory harmony in which sibilants assimilate in [anterior] to the input value of the rightmost sibilant in the word. As seen in Table 1, for an input with two distinct sibilants (/a Jasa/), the optimal output (asasa) has two identical sibilants:

\begin{tabular}{|r|l|c|c|c|}
\hline & /aJasa/ & $\begin{array}{c}\text { CoRR-SS, } \\
\text { ID-SS-ant }\end{array}$ & $\begin{array}{c}\text { ID-IO-ant } \\
\text { (x left-index })\end{array}$ & \\
\hline \hline & & 2 & 1 & $\mathcal{H}$ \\
\hline \hline a. & ajasa & -1 & & -2 \\
\hline b. & asasa & & $-1 \times 1=\mathbf{- 1}$ & -1 \\
\hline c. & ajaja & & $-1 \times 2=\mathbf{- 2}$ & -2 \\
\hline
\end{tabular}

Table 1: Regressive sibilant harmony

In this tableau, assimilation is enforced by the top-weighted correspondence constraint complex, which requires co-existing sibilants in the same word to correspond (CORR-SS) and be identical (ID-SS-ant). ${ }^{1}$ The

\footnotetext{
* We are grateful to audience at AMP and at the UC Berkeley Phonetics and Phonology Forum, and to Brian Smith, for feedback and challenging questions.

1 Following other recent work in surface correspondence theory, we lump together correspondence and identity 
direction of assimilation (anticipatory, as here, vs. perseverative) is due to the distance-based penalty scaling of the faithfulness constraint ID-IO-ant. This constraint is violated when an input sibilant surfaces with an unfaithful value for [ant]. The farther from the indexed edge (in this case, left) the sibilant is, the higher its penalty. Each violation of left-indexed ID-IO-ant is scaled linearly by its distance from the left edge; thus the multiplier for violations in the $n^{\text {th }}$ sibilant, counting from the left, is $n$. We assume that distance is measured on a relevant projection, in this case, the projection of sibilants, with the lowest value being 1 . (On the use of projections in Correspondence Theory, see e.g. Hansson (2014); cf. Legendre et al. (2006)). Thus the first sibilant in a word is subject to a scaling factor of 1 ; the second sibilant in a word is subject to a scaling factor of 2, etc. In this tableau, distance-based penalty scaling is what causes winning candidate (b) to outperform losing candidate (c). Both contain exactly one unfaithful sibilant, but its distance from the left edge results in differential penalty scores.

A graphical representation of the penalty scaling used in the toy example in Table 1 is given in Figure 1:

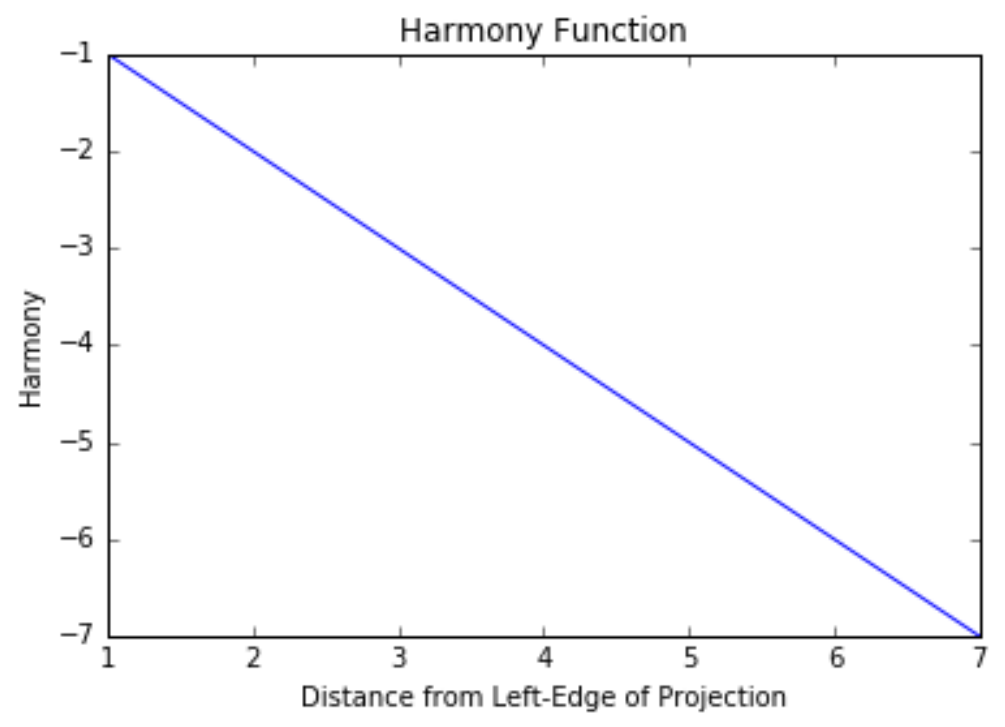

Figure 1: Linear distance scaling 3.2 .

Penalty scaling can be linear, as in Figure 1, or exponential; we introduce non-linear scaling in section

2.2 Other scalar constraint weighting proposals Distance-based penalty scaling involves increases to penalties in individual cells, and thus connects with other recent work proposing cell multipliers. Zymet (2014) proposes non-linear penalty scaling to account for trigger-target distance preconditions in harmony. Boersma \& Pater (2016) use constraint scaling factors to achieve 'noisy' effects in Harmonic Grammar; Hayes (2016) proposes an implementation in which individual violations instead are randomly scaled. A number of researchers have used scalar constraint weighting factors to capture language-specific lexical and morphological stratal effects (e.g., Shih \& Inkelas (2015); Hsu \& Jesney (2016); McPherson \& Hayes (2016); Stanton (2016)).

For example, in an analysis of stratal effects in loanword adaptation in French, Hsu \& Jesney (2016) define six lexical strata, focusing on the marked structures $*_{I} V$ and $* t \int$. In strata 1-2, both illicit sequences are repaired, reflecting native French phonotactics. In strata 3-5, violations of $*_{I} V$ are repaired, but violations of *t $\int$ are not. Stratum 6 repairs violations of neither constraint; $I V$ and $t \int$ are produced faithfully in loanwords of this stratum. Hsu \& Jesney propose to scale faithfulness violations differently in each stratum of the language. This amounts to adjusting the weight of Faith. A graphical depiction of the result of scaling Faithfulness penalties from the 'core' stratum (lowest value $=1$ ) to the 'periphery' stratum (highest value = constraints; see e.g., Hansson (2014); Walker (2015); Shih \& Inkelas (forthcoming). This space-saving decision is immaterial to the arguments in this paper. 
6) is given in Figure 2. As seen, the weight of Faith overtakes that of its fellow markedness constraints in certain strata.

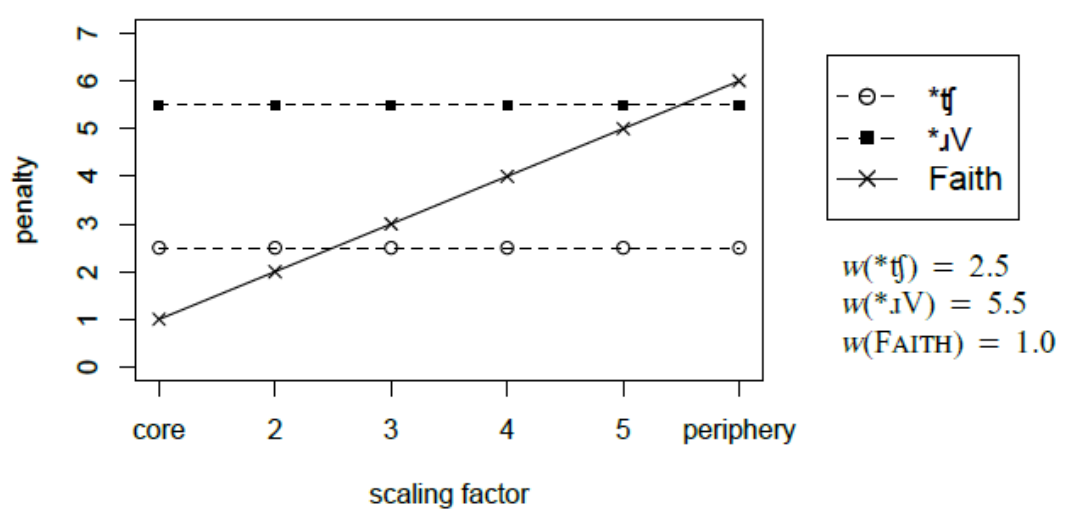

Figure 2: Linear stratal scaling

2.3 Case study: distance-based scaling in Nkore-Kiga Having seen a simple formal illustration of distance-based scaling, we now turn to a more complex real-life example of anticipatory sibilant harmony in Nkore-Kiga (Bantu, Uganda). This language is well known for a pattern of harmony which is both directional and opaque, and in which the trigger of harmony is not an input value but one governed by local markedness constraints (Hansson, 2001; Bennett \& Pulleyblank) (to appear))

In Nkore-Kiga, [s] and [ $]$ ] are in complementary distribution: [s] occurs before [i], and [f] occurs before $[\mathrm{e}, \mathrm{a}, \mathrm{u}, \mathrm{o}]$ ("S-MARKEDNESS"). This distribution is disrupted when there is more than one sibilant in a word. In that case, all sibilants must agree in [anterior]. Importantly, the harmony process is governed by the surface form of the rightmost sibilant.

\begin{tabular}{|l|l|l|l|l|l|}
\hline a. & /-Sa:S-ire/ & sa:sire & $(*$ fa:sire $)$ & 'be in pain (perf.)' & (cf. fa:f- $a$ 'be in pain') \\
\hline b. & /-Si:S-a/ & fi:fa & $($ (si:fa) & 'do wrong, sin' & \\
\hline
\end{tabular}

Table 2: Nkore-Kiga sibilant harmony

Nkore-Kiga presents a challenge to existing approaches to directionality. While the directionality effect is simple to describe, it has proved challenging to implement. Conventional AGREE or correspondence constraints can compel two sibilants to correspond and agree. However, they cannot determine which one should depart from local markedness requirements in order to agree with the other. The alternation is allophonic and symmetric; it does not favor one value of [anterior] over the other. Nor is special faithfulness, of the conventional sort, applicable. The trigger consonant is neither necessarily faithful to input, nor does it consistently occupy a designated prominent position (such as word-final, or onset of the stressed syllable) which would make it possible to use a positional faithfulness or positional markedess analysis, à la Beckman (1997) or Zoll (1997). Rather, it is the case that the consonant which obeys local markedness phonotactic constraints, and which 'triggers' agreement on the part of the other consonant, is the one which is relatively closest to the right edge.

Hansson (2001) sought to account for this directionality effect through the use of targeted constraints, a mechanism no longer in wide use (see e.g. McCarthy (2002)). Bennett \& Pulleyblank (to appear) propose to condition the alternation morphologically, avoiding a phonological account altogether.

We offer a straightforward phonological solution: distance-based penalty scaling for the local markedness constraints *sa and *ji.

In the tableau in Table 3, the distance-scaled constraint is S-MARKEDNESS, violated by a sibilant whose value for [anterior] is inconsistent with what the following vowel demands. S-MARKEDNESS is indexed to the left edge, meaning that a sibilant later in a word has a higher penalty for violating S-MARKEDNESS than does a sibilant earlier in the word. All three candidates in Table 3 possess two sibilants. The first sibilant has a distance-based penalty scaling factor of 1; the second has a scaling factor of 2. Candidate (a) has two different sibilants, each conforming to S-MARKEDNESS in its local context, but violating the high-weighted harmony complex; it fails. Candidates (b) and (c) have sibilants which 


\begin{tabular}{|r|l|c|c|c|}
\hline & /-Sa:S-ire/ & $\begin{array}{c}\text { CORR-SS, } \\
\text { ID-CC-ant }\end{array}$ & $\begin{array}{c}\text { S-MARKEDNESS } \\
\text { (x left-index) }\end{array}$ & \\
\hline \hline & & 2 & 1 & $\mathcal{H}$ \\
\hline \hline a. & Jarsire & -1 & & -2 \\
\hline b. & sarsire & & $(-1 \times 1)=\mathbf{- 1}$ & -1 \\
\hline c. & farfire & & $(-1 \times 2)=\mathbf{- 2}$ & -2 \\
\hline
\end{tabular}

Table 3: Distance-scaled analysis of Nkore-Kiga sibilant harmony

agree with one another, satisfying harmony constraints, but each violating S-MARKEDNESS once. The offending sibilant in candidate (c) is the second sibilant, thus farther from the left edge, and its violation of S-MARKEDNESS is thus higher than that of winning candidate (b).

In sum, distance-based penalty scaling for a markedness constraint achieves directional harmony in Nkore-Kiga without encountering the problems suffered by past accounts which rely on absolute position or faithfulness to generate directionality effect.

\section{Edge Effects}

3.1 Linear Scaling In addition to directional harmony phenomena, we also extend our distance-based penalty scaling approach in the analysis of edge effects, such as those involving 'opposite-edge effects' (see e.g., Zoll (1997); Alber (2005)). Opposite-edge effects involve the apparent conflict between forces which motivate alignment or sensitivity towards one end of a domain, and forces which motivate towards the opposite edge. For example, a well known case of such an opposite edge effect is the case of palatalization in Japanese mimetic vocabulary. To overview the conflicting directionality facts, ${ }^{2}$ coronals in coronal-noncoronal sequences are palatalized. If there is more than one coronal, the rightmost coronal is palatalized. However, if there are no coronals, the left-most noncoronal is palatalized. This distinction between left-preference for noncoronals and right-preference for coronals illustrates the conflicting directionality nature of opposite-edge effects.

Such effects can be modeled effectively through distance-based penalty scaling. In the case of the Japanese palatalization, we model this opposite-edge behavior through the interaction between two scaled constraints: $* \mathrm{p}^{j}$ (markedness constraint dispreferring non-coronal palatalization) and an ID faithfulness constraint. The constraint $* \mathrm{p}^{j}$ is left-indexing, meaning that its violations are scaled based on distance from the left edge of the projection. This captures the pressure to palatalize non-coronals closer to the beginning of the word. The ID constraint, however, is right-indexing, meaning that violations of this faithfulness constraint become more severe as the locus of violation moves away from the right edge of the word. The application of these constraints is presented in Table 4.

\begin{tabular}{|r|c|c|c|c|c|}
\hline & /ptptp/ & HAVEPAL & $\begin{array}{c}* \mathrm{p}^{j} \\
(\text { left-index) }\end{array}$ & $\begin{array}{c}\text { ID } \\
(\text { right-index })\end{array}$ & \\
\hline \hline & & 7 & 2 & 1 & $\mathcal{H}$ \\
\hline \hline $\mathrm{a}$. & $\mathrm{ptptp}$ & -1 & & & -7 \\
\hline b. & $\mathrm{ptptp}^{j}$ & & $(-1 \times 3)=\mathbf{- 3}$ & $(-1 \times 1)=\mathbf{- 1}$ & -7 \\
\hline c. & $\mathrm{ptpt}^{j} \mathrm{p}$ & & & $(-1 \times 2)=\mathbf{- 2}$ & -2 \\
\hline $\mathrm{d}$. & $\mathrm{pt}^{j} \mathrm{ptp}$ & & & $(-1 \times 4)=\mathbf{- 4}$ & -4 \\
\hline $\mathrm{e}$. & $\mathrm{p}^{j} \mathrm{tptp}$ & & $(-1 \times 1)=\mathbf{- 1}$ & $(-1 \times 5)=\mathbf{- 5}$ & -7 \\
\hline
\end{tabular}

Table 4: Japanese Mimetic Palatalization: Coronals

In Table 4, the scaling of the ID constraint allows us to adjudicate between candidates (c) and (d). Without scaling, both of these candidates would have one violation of ID and would be tied. With scaling, the violations for the right-most

\footnotetext{
2 Setting aside complications of $/ \mathrm{r} /$.
} 
palatalized coronal (c) are less than its competitor (d), leading to its correct generation.

The interaction between the left-indexed $* \mathrm{p}^{j}$ and the right-indexed ID constraints is visible in the analysis of words without coronals, as in Table 5. Although the violation of ID in the winning candidate (c) through palatalization of the initial non-coronal is greater than its competitors, this form has the smallest violation of the left-indexed $* \mathrm{p}^{j}$ constraint. The right-indexed effect of the ID constraint is only visible in cases in which ${ }^{*} \mathrm{p}^{j}$ does not apply (i.e., in words containing coronals).

\begin{tabular}{|r|l|c|c|c|c|}
\hline & $/ \mathrm{pppp} /$ & HAVEPAL & $\begin{array}{c}* \mathrm{p}^{j} \\
(\text { left-index })\end{array}$ & $\begin{array}{c}\text { ID } \\
(\text { right-index })\end{array}$ & \\
\hline \hline & & 7 & 2 & 1 & $\mathcal{H}$ \\
\hline \hline a. & $\mathrm{pppp}$ & -1 & & & -7 \\
\hline b. & $\mathrm{pppp}^{j}$ & & $(-1 \times 4)=\mathbf{- 4}$ & $(-1 \times 1)=\mathbf{- 1}$ & -9 \\
\hline c. & $\mathrm{p}^{j} \mathrm{ppp}$ & & $(-1 \times 1)=\mathbf{- 1}$ & $(-1 \times 4)=\mathbf{- 4}$ & -6 \\
\hline
\end{tabular}

Table 5: Japanese Mimetic Palatalization: No Coronals

A similar case of conflicting directionality in another domain is Selkup stress. In this system, as described by Zoll (1997), rightmost heavy syllables receive stress. However, if there are no heavy syllables, the leftmost light syllable receives stress. This behavior is exemplified in (1).

$$
\text { Selkup stress examples from Halle \& Clements (1983) }
$$

a. /u:'co:mit/ - 'we work' (rightmost heavy)

b. /'karman/ - 'pocket' (leftmost light)

The parallels between this phenomenon and the case of Japanese mimetic palatalization are clear. Our analysis of the Selkup stress system also involves a right-index ID constraint and includes a left-indexed constraint motivated against stressed light-syllables. The left-indexed nature of this constraint ensures that, if a light-syllable must be stressed, the least egregious violation of this constraint is the leftmost light. The application of these constraints is shown in Tables 6 and 7 .

\begin{tabular}{|r|l|c|c|c|c|}
\hline & /HLHL/ & HAVESTRESS & $\begin{array}{c}* \dot{L} \\
\text { (left-index) }\end{array}$ & $\begin{array}{c}\text { ID } \\
\text { (right-index) }\end{array}$ & \\
\hline \hline & & 7 & 2 & 1 & $\mathcal{H}$ \\
\hline \hline a. & HLHL & -1 & & & -7 \\
\hline b. & HLHĹ & & $(-1 \times 4)=\mathbf{- 4}$ & $(-1 \times 1)=\mathbf{- 1}$ & -9 \\
\hline c. & HLH́L & & & $(-1 \times 2)=\mathbf{- 2}$ & -2 \\
\hline d. & HĹHL & & $(-1 \times 2)=\mathbf{- 2}$ & $(-1 \times 3)=\mathbf{- 3}$ & -7 \\
\hline e. & H́LHL & & & $(-1 \times 4)=\mathbf{- 4}$ & -4 \\
\hline
\end{tabular}

Table 6: Selkup Stress: Heavy and Light Syllables

3.2 Non-Linear Scaling and Absolute Edge Effects In the opposite-edge examples discussed in Section 3.1, we saw the application of linear distance-based penalty scaling. This model may also be extended to the analysis of absolute edge effects through the use of non-linear scaling functions. As a test case, we present an analysis of the formation of various impersonal verb forms from Chaha. This verbal paradigm demonstrates both gradient edge effects as well as absolute edge tropism.

Chaha exhibits robust labialization and palatalization processes in various areas of the grammar ((Leslau, 1967; McCarthy, 1983; Banksira, 2000, 2013; Rose, 2007). Depending on the exact paradigm in question, these processes may be absolute edge effects ('palatalize the final root consonant') or gradient edge effects ('labialize the right-most root consonant'). In the impersonal paradigm, both labialization and palatalization are active. Palatalization in the impersonal is an absolute edge effect: the final coronal obstruent of the root is palatalized. In contrast, labialization is a gradient edge 


\begin{tabular}{|r|l|c|c|c|c|}
\hline & /LLLL/ & HAVESTRESS & $\begin{array}{c}* \mathrm{~L} \\
\text { (left-index) }\end{array}$ & $\begin{array}{c}\text { ID } \\
\text { (right-index) }\end{array}$ & \\
\hline \hline & & 7 & 2 & 1 & $\mathcal{H}$ \\
\hline \hline a. & LLLL & -1 & & & -7 \\
\hline b. & LLLĹ & & $(-1 \times 4)=\mathbf{- 4}$ & $(-1 \times 1)=\mathbf{- 1}$ & -9 \\
\hline c. & LLĹL & & $(-1 \times 3)=\mathbf{- 3}$ & $(-1 \times 2)=\mathbf{- 2}$ & -8 \\
\hline d. & LĹLL & & $(-1 \times 2)=\mathbf{- 2}$ & $(-1 \times 3)=\mathbf{- 3}$ & -7 \\
\hline a a. & ĹLLL & & $(-1 \times 1)=\mathbf{- 1}$ & $(-1 \times 4)=\mathbf{- 4}$ & -6 \\
\hline
\end{tabular}

Table 7: Selkup Stress: Only Light Syllables

effect in the impersonal: (unless already patalalized) the rightmost velar or labial consonant is labialized. These data, taken from Banksira (2013:164), are analyzed in Table 8.

Under our schematized analysis, palatalization and labialization are enforced by the constraints HAVEPAL and HAVELAB, respectively. These faithfulness constraints are opposed by markedness constraints against palatalized $\left({ }^{*} \mathrm{C}^{y}\right)$ and labialized segments $\left({ }^{*} \mathrm{C}^{w}\right)$. As in the Selkup examples above, these markedness constraints are scaled and indexed to edges of the verb root. In this case, both constraints are right-indexed, meaning that violations further from the right edge of the root accrue greater harmony penalties.

The form for "open" is subject to both palatalization and labialization; candidate 3 is the attested winner. The form for "ahead", however, demonstrates that palatalization in the impersonal does not apply when a coronal obstruent is not root-final, even if a coronal obstruent appears earlier in the root. Candidate 08 is chosen over candidate 07 which has palatalization on a non-root-final coronal obstruent.

\begin{tabular}{|c|c|c|c|c|c|c|}
\hline “open" & kift-im & $\begin{array}{l}\text { HAVE } \\
\text { LAB. }\end{array}$ & $\begin{array}{l}\text { HAVE } \\
\text { PAL. }\end{array}$ & $\begin{array}{c}* \mathrm{C}^{y} \\
\text { (R-index; exp.) }\end{array}$ & $\begin{array}{c}* \mathrm{C}^{w} \\
\text { (R-index; linear) }\end{array}$ & \\
\hline & & $w$ & $x$ & $y$ & $z$ & $\mathcal{H}$ \\
\hline 01 & kift-im & -1 & -1 & 0 & 0 & \\
\hline 02 & kifč-im & -1 & 0 & $-1 * f(1)$ & 0 & \\
\hline 唵 03 & $\mathrm{kif}^{\mathrm{w}}{ }^{\mathrm{c}} \check{\mathrm{c}}$-im & 0 & 0 & $-1 * f(1)$ & $-1 * g(3)$ & \\
\hline 04 & kif ${ }^{\mathrm{w}} \mathrm{t}-\mathrm{im}$ & 0 & -1 & 0 & $-1 * g(3)$ & \\
\hline "ahead" & bidər-im & $\begin{array}{l}\text { HAVE } \\
\text { LAB. }\end{array}$ & $\begin{array}{l}\text { HAVE } \\
\text { PAL. }\end{array}$ & $\begin{array}{c}* \mathrm{C}^{y} \\
\text { (R-index; exp.) }\end{array}$ & $\begin{array}{c}* \mathrm{C}^{w} \\
\text { (R-index; linear) }\end{array}$ & \\
\hline & & $w$ & $x$ & $y$ & $z$ & $\mathcal{H}$ \\
\hline 05 & bidər-im & -1 & -1 & 0 & 0 & \\
\hline 06 & bid $^{\mathrm{y}}$ ər-im & -1 & 0 & $-1 * f(3)$ & 0 & \\
\hline 07 & $\mathrm{~b}^{\mathrm{w}} \mathrm{id}^{\mathrm{y}}$ วr-im & 0 & 0 & $-1 * f(3)$ & $-1 * g(5)$ & \\
\hline 喓 08 & $\mathrm{~b}^{\mathrm{w}}$ idər-im & 0 & -1 & 0 & $-1 * g(5)$ & \\
\hline
\end{tabular}

Table 8: Chaha Impersonal Labialization and Palatalization

Where $f(n)=a^{n}$ and $g(n)=b * n$.

The system of constraint weights and scaling factors which we solve for must capture both edge-gradient as well as edge-absolute behavior. Specifically, the system must ensure that a violation of ${ }^{*} \mathrm{C}^{y}$ is permitted root-finally, but not earlier in the root.

While in previous examples it has sufficed to present schematized analyses with hand-chosen constraint weights, introducing non-linear scaling motivates the use of methods for mathematically solving for constraint weights and scaling factors. We follow the methods outlined in Potts et al. (2010) and use linear programming to test for viable solutions. Briefly, this approach treats each Winning-Losing candidate pair as an inequality that constrains the space of possible model parameters. Each additional candidate pair and each additional input form add further restrictions to the space of possible solutions. If winning candidates from two input forms are logically incompatible (say one requires constraint 
A's weight to be greater than constraint B, but another requires the reverse), then this non-solution is correctly identified.

Neatly delimiting the space of possible solutions to a system like this is often computationally intractable. Instead, we use minimization techniques to solve these systems of inequalities. Specifically, we use the Sequential Least Squares algorithm as implemented in Python's scipy-optimize package. Given that this technique is a minimization algorithm, the constraint weights returned are often quite small, and the harmony differences between candidates also are quite small. We choose to utilize these values for computational convenience and note that there are countless other possible solutions in the space of solutions which also solve this system; the exact numerical values chosen for this particular solution are of no particular interest.

\begin{tabular}{|c|c|c|c|c|c|c|}
\hline "open" & kift-im & $\begin{array}{l}\text { HAVE } \\
\text { LAB. }\end{array}$ & $\begin{array}{l}\text { HAVE } \\
\text { PAL. }\end{array}$ & $\begin{array}{c}{ }^{*} \mathrm{C}^{y} \\
\text { (R-index; exp.) }\end{array}$ & $\begin{array}{c}{ }^{*} \mathrm{C}^{w} \\
\text { (R-index; linear) }\end{array}$ & \\
\hline & & $w$ & $x$ & $y$ & $z$ & $\mathcal{H}$ \\
\hline 01 & kift-im & -1 & -1 & 0 & 0 & -6.000014 \\
\hline 02 & kifč-im & -1 & 0 & $-1 * f(1)$ & 0 & -6.000013 \\
\hline 实 03 & $\mathrm{kif}^{\mathrm{w}} \mathrm{c}$-im & 0 & 0 & $-1 * f(1)$ & $-1^{*} \mathrm{~g}(3)$ & -4.000008 \\
\hline 04 & kiff ${ }^{w}$ t-im & 0 & -1 & 0 & $-1^{*} \mathrm{~g}(3)$ & -4.000009 \\
\hline
\end{tabular}

\begin{tabular}{|c|c|c|c|c|c|c|}
\hline "ahead" & bidər-im & $\begin{array}{l}\text { HAVE } \\
\text { LAB. }\end{array}$ & $\begin{array}{l}\text { HAVE } \\
\text { PAL. }\end{array}$ & $\begin{array}{c}* \mathrm{C}^{y} \\
\text { (R-index; exp.) }\end{array}$ & $\begin{array}{c}{ }^{*} \mathrm{C}^{w} \\
\text { (R-index; linear) }\end{array}$ & \\
\hline & & $w$ & $x$ & $y$ & $z$ & $\mathcal{H}$ \\
\hline 05 & bidər-im & -1 & -1 & 0 & 0 & -6.000014 \\
\hline 06 & bid $^{y}$ วr-im & -1 & 0 & $-1 * f(3)$ & 0 & -6.000015 \\
\hline 07 & $\mathrm{~b}^{\mathrm{w}} \dot{\mathrm{id}}^{\mathrm{y}}$ ər-im & 0 & 0 & $-1 * f(3)$ & $-1 * g(5)$ & -6.000014 \\
\hline 实 08 & $\mathrm{~b}^{\mathrm{w}} \mathrm{id} \mathrm{id}$-im & 0 & -1 & 0 & $-1 * g(5)$ & -6.000013 \\
\hline
\end{tabular}

Table 9: Chaha Impersonal Labialization and Palatalization ${ }^{3}$

After optimization, the system in Table 8 is shown to be well-formed and logically possible. The resulting constraint weights, scaling factors, and harmonies are presented in Table 9. After solving for constraint weights and scaling factors, the interplay between the weight of HavePal and scaling of $* \mathrm{C}^{y}$ is clear, as can be observed in Figure $3 \mathrm{a}$ and its crosssection in Figure 3b. The absolute-edge effect exhibited by Chaha impersonals is successfully generated under this system, as the violation at the rightmost consonant $(\mathrm{x}=1)$ is less than the weight of HavePal (red line), while a violation at subsequent consonants $(\mathrm{x}>=2)$ is greater than (below) the weight of HavePal.

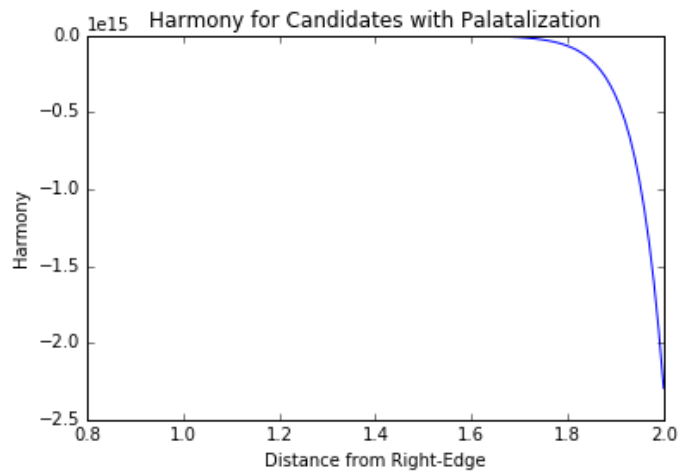

(a) Full view

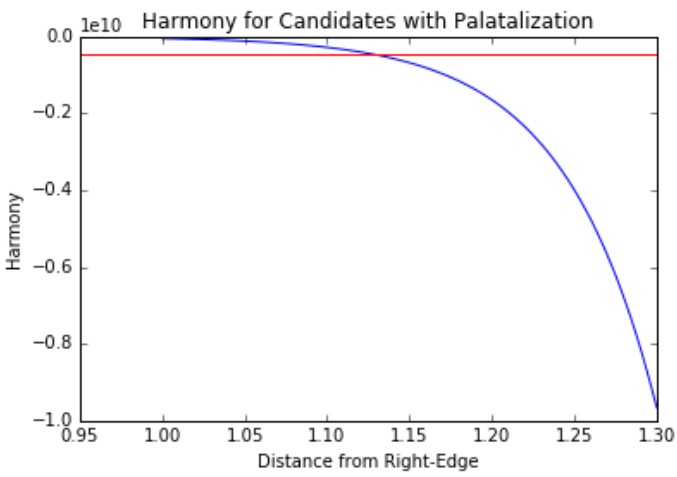

(b) Cross-section

Figure 3: Chaha Absolute Edge Palatalization

\footnotetext{
3 Where $w=5.0000109999999554, x=1.0000029999999698, y=1.0000009999999893, z=1.0000009999999853$, $f(n)=1.000000999999981^{n}$, and $g(n)=1.0000010000000079 * n$.
} 


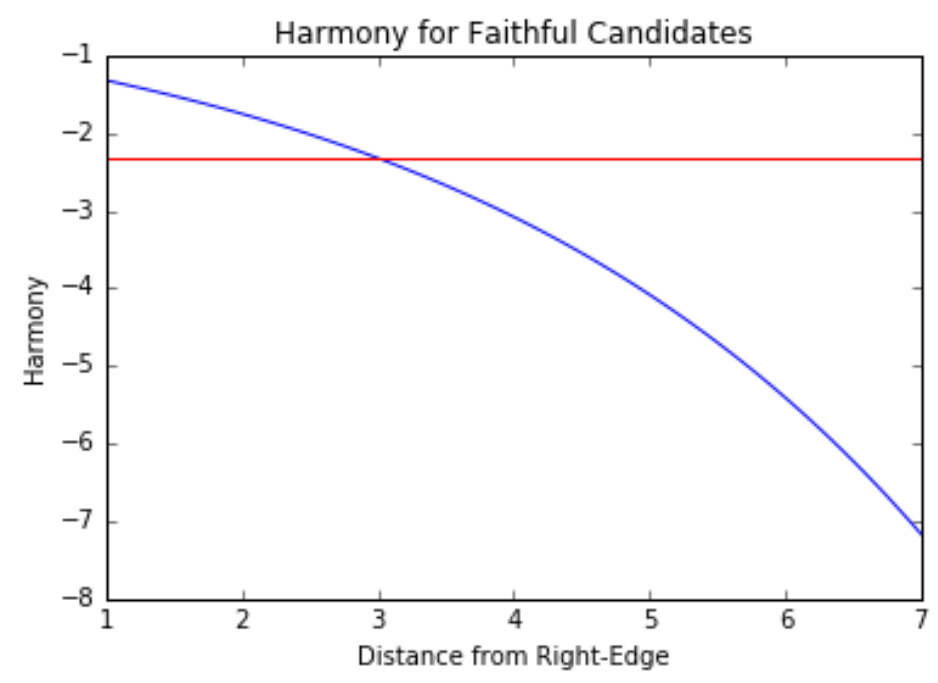

Figure 4: Harmonic Bounding of Scaled Constraint

\section{Windows}

As we've seen, distance-based scaling can efficiently model a variety of directional effects. The nonlinear function used for in the derivation of Chaha absolute-edge effects can be extended to the analysis of window phenomena. In what follows, we present an analysis of the most well-known of these window phenomena: metrical stress windows. Under our model, these metrical stress windows are captured by the same mechanism as absolute-edge effects. The difference between these two phenomena lies only in the inflection point of the scaling function.

For a language with metrical windows, primary stress may only fall within a certain size syllable window at an edge of a word. For example, the system of Macedonian (Kager, 2012:1457) has a three-syllable final window.

$$
\begin{array}{ll}
\text { a. } & \text { ad.vo.'kat - 'lawyer' } \\
\text { b. ad.vo.'ka.ti - 'lawyers' } \\
\text { c. ad.vo.'ka.ti.te - 'the lawyers' }
\end{array}
$$

$$
\begin{aligned}
& \text { a. kon.zu.'ma.tor - 'consumer' } \\
& \text { b. kon.zu.ma.'to.ri.te - 'the consumers' (cf. *kon.zu.'ma.to.ri.te) }
\end{aligned}
$$

In (2), we can observe that the underlying stress will remain aligned to its original syllable despite the addition of inflectional suffixes. However, a stress outside of a final three-syllable window is not permitted. If the underlying stressed syllable would fall outside of this window with the addition of suffixes, the attested repair is to align stress with the left-most syllable in the window, the antepenult, as in (3).

Let us consider how we might model such a language with a three-syllable word-final window where the default position is the antepenultimate syllable using distance-based penalty scaling. Descriptively, we want the system to realize stress faithfully if it falls within a three-syllable word-final window. Otherwise, stress should default to the left edge of the window (antepenultimate).

To model this phenomenon we will use a right-indexed scaled markedness constraint, *STRESS-R, which penalizes stressed syllables. The right-indexed nature of this constraint penalizes stressed syllables more heavily as they move further from the right-edge of the word.

Given this single distance-scaled constraint, a system such as Macedonian cannot be generated. This is because the scaled constraint prefers the candidate with stress on the right-most syllable. Candidates with default stress further left, such as our desired antepenultimate system, are harmonically bounded. Changing the scaling factor would allow for different cutoffs of Faith (and thus change the size of the window), but would not create a more optimal candidate for repair anywhere else. This is demonstrated visually in Figure 4, in which the harmony penalty associated with various candidates is shown. Regardless of the scaling factor associated with this function, the penalty for a stressed antepenultimate will be greater than for a stressed final syllable. 
In order to generate a system which still respects the metrical stress window but prefers (and repairs to) antepenultimate stress, we introduce a second left-indexed scaled constraint: *STRESS-L (cf. ALIGN-L). This constraint behaves identically to ${ }^{*}$ STRESS-R, except that it penalizes stressed syllables more heavily the further they are from the left edge of the word. The combination of these two competing forces generates default antepenultimate stress while respecting the window.

\begin{tabular}{|rc||c|c|c|}
\hline & SSSSSSS & FAITH & *STRESS-R & *STRESS-L \\
\hline \hline 实 01 & ssssssS & 0 & $-1 * \mathrm{f}(1)$ & $-1 * \mathrm{~g}(7)$ \\
\hline 02 & Sssssss & -1 & $-1 * \mathrm{f}(7)$ & $-1 * \mathrm{~g}(1)$ \\
\hline 03 & sssssSs & -1 & $-1 * \mathrm{f}(2)$ & $-1 * \mathrm{~g}(6)$ \\
\hline 04 & ssssSss & -1 & $-1 * \mathrm{f}(3)$ & $-1 * \mathrm{~g}(5)$ \\
\hline 05 & sssSsss & -1 & $-1 * \mathrm{f}(4)$ & $-1 * \mathrm{~g}(4)$ \\
\hline
\end{tabular}

\begin{tabular}{|rc||c|c|c|}
\hline & SSSSSss & FAITH & *STRESS-R & $*$ STRESS-L \\
\hline \hline 06 & ssssss & -1 & $-1 * \mathrm{f}(1)$ & $-1 * \mathrm{~g}(7)$ \\
\hline 07 & Sssssss & -1 & $-1 * \mathrm{f}(7)$ & $-1 * \mathrm{~g}(1)$ \\
\hline 08 & sssssSs & -1 & $-1 * \mathrm{f}(2)$ & $-1 * \mathrm{~g}(6)$ \\
\hline 09 & ssssSss & 0 & $-1 * \mathrm{f}(3)$ & $-1 * \mathrm{~g}(5)$ \\
\hline 10 & sssSsss & -1 & $-1 * \mathrm{f}(4)$ & $-1 * \mathrm{~g}(4)$ \\
\hline
\end{tabular}

\begin{tabular}{|rc||c|c|c|}
\hline & SSSSSSS & FAITH & *STRESS-R & *STRESS-L \\
\hline \hline 11 & ssssssS & -1 & $-1 * \mathrm{f}(1)$ & $-1 * \mathrm{~g}(7)$ \\
\hline 12 & Sssssss & -1 & $-1 * \mathrm{f}(7)$ & $-1 * \mathrm{~g}(1)$ \\
\hline 13 & sssssSs & -1 & $-1 * \mathrm{f}(2)$ & $-1 * \mathrm{~g}(6)$ \\
\hline 14 & ssssSss & -1 & $-1 * \mathrm{f}(3)$ & $-1 * \mathrm{~g}(5)$ \\
\hline 15 & sssSsss & 0 & $-1 * \mathrm{f}(4)$ & $-1 * \mathrm{~g}(4)$ \\
\hline
\end{tabular}

Table 10: Representation of Macedonian-Type System Where $f(n)=\alpha^{n}$ and $g(n)=\beta^{n}$

After solving the system of constraint inequalities in Table 11, the intuition behind the constraint interaction can be visualized, as in Figure 5.

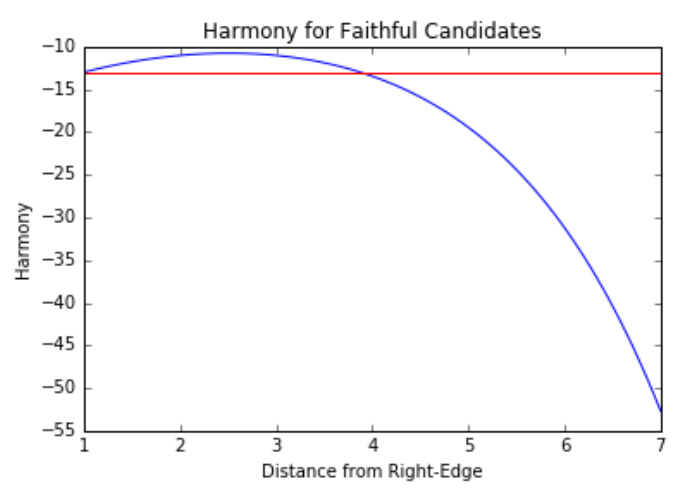

(a) Full view

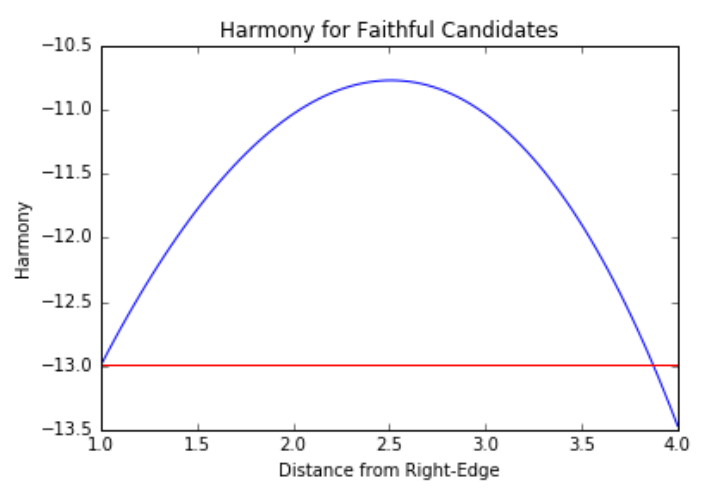

(b) Cross-section

Figure 5: Harmony Function for Macedonian-Type System

The combined penalties associated with *STRESS-L and *STRESS-R serve to create a harmony maximum closest to the antepenultimate syllable. ${ }^{5}$ In both Figure $5 \mathrm{a}$ and the cross-section in Figure $5 \mathrm{~b}$, the harmony penalty associated

\footnotetext{
${ }^{4}$ Where $x=1.9594915238194803, y=1.0000010002276869, z=1.0000010022518417, f(n)=$ $1.7548224302216895^{n}$, and $g(n)=1.4128649746767603^{n}$

5 The actual minimum is slightly more than 2.5 . This is due to our solving the system of inequalities through continuous,
} 


\begin{tabular}{|c|c|c|c|c|c|}
\hline & \multirow[t]{2}{*}{ SSSSSSS } & FAITH & *STRESS-R & *STRESS-L & \\
\hline & & $x$ & $y$ & $z$ & $\mathcal{H}$ \\
\hline 实 01 & ssssssS & 0 & $-1 * f(1)$ & $-1 * g(7)$ & -12.993238 \\
\hline 02 & Sssssss & -1 & $-1 * f(7)$ & $-1 * g(1)$ & -54.615136 \\
\hline 03 & sssssSs & -1 & $-1 * f(2)$ & $-1 * g(6)$ & -12.993240 \\
\hline 04 & SsssSss & -1 & $-1 * f(3)$ & $-1 * g(5)$ & -12.993239 \\
\hline 05 & SssSsss & -1 & $-1 * f(4)$ & $-1 * g(4)$ & -15.426984 \\
\hline
\end{tabular}

\begin{tabular}{|rl||c|c|c||c|}
\hline & SSSSSSS & FAITH & *STRESS-R & *STRESS-L & \\
\hline \hline & & $x$ & $y$ & $z$ & $\mathcal{H}$ \\
\hline \hline 06 & ssssssS & -1 & $-1 * \mathrm{f}(1)$ & $-1 * \mathrm{~g}(7)$ & -14.952730 \\
\hline 07 & Ssssss & -1 & $-1 * \mathrm{f}(7)$ & $-1 * \mathrm{~g}(1)$ & -54.615136 \\
\hline 08 & sssssSs & -1 & $-1 * \mathrm{f}(2)$ & $-1 * \mathrm{~g}(6)$ & -12.993240 \\
\hline 09 & ssssSss & 0 & $-1 * \mathrm{f}(3)$ & $-1 * \mathrm{~g}(5)$ & -12.993239 \\
\hline 10 & sssSsss & -1 & $-1 * \mathrm{f}(4)$ & $-1 * \mathrm{~g}(4)$ & -13.467492 \\
\hline
\end{tabular}

\begin{tabular}{|c|c|c|c|c|c|}
\hline & SSSSsSS & FAITH & *STRESS-R & *STRESS-L & \\
\hline & & $x$ & $y$ & $z$ & $\mathcal{H}$ \\
\hline 11 & ssssssS & -1 & $-1 * f(1)$ & $-1 * g(7)$ & -14.952730 \\
\hline 12 & Sssssss & -1 & $-1 * f(7)$ & $-1 * g(1)$ & -54.615136 \\
\hline 13 & sssssSs & -1 & $-1 * f(2)$ & $-1 * g(6)$ & -12.993240 \\
\hline 喓 14 & ssssSss & -1 & $-1 * f(3)$ & $-1 * g(5)$ & -11.033748 \\
\hline 15 & sssSsss & 0 & $-1 * f(4)$ & $-1 * g(4)$ & -15.426984 \\
\hline
\end{tabular}

Table 11: Solution for Macedonian-Type System ${ }^{4}$

with FAITH (red line) enforces the three-syllable window; stress will only default to the antepenultimate stress when its combined violations of $*$ STRESS-R and $*$ STRESS-L exceeds this threshold.

\section{Discussion}

This brief study has shown that by appealing to both linear and nonlinear distance-based penalty scaling functions, we can capture both bounded and unbounded distance effects in directional harmony. The same mechanism can generate window-based distributions of phonological properties within words, linking together window effects with traditional directional phenomena in a manner reminiscent of McCarthy's (2003) 'bounded gradience.'

Distance-based penalty scaling has clear implications beyond the edge effects discussed in this paper. For example, distance-based penalty scaling can extend to word-internal interactions between segments at a particular distance from one another (see e.g. Zymet (2014)). We believe that the approach will also extend naturally to word-internal windowtype effects, such as bounded phonotactic restrictions on assimilation or dissimilation (see e.g., Bennett (2013)), as well as to 'trough' effects of the type documented by Hyman (1998), in which harmony applies only within a medial window.

Many open questions remain, including the variety of nonlinear functions that may be used in the theory, the size of the windows that such functions can define, and the typological predictions generated by allowing any number of constraints to be distance-scaled. Much future work is needed to answer these questions.

\section{References}

Alber, Birgit (2005). Clash, lapse and directionality. Natural Language and Linguistic Theory 23.

rather than integer-based minimization algorithms. Importantly, the antepenultimate candidate $(x=3)$ is the closest candidate to this minimum. 
Bakovic, Eric \& Sharon Rose (2014). Directionality in distance effects. UC Berkeley Phonology Lab 2014 Annual Report: ABCConference 63-73.

Banksira, Degif Petros (2000). Sound mutations: the morphophonology of Chaha. John Benjamins, Amsterdam/Philadelphia.

Banksira, Degif Petros (2013). Chaha labialization and palatalization as coalescence. Brill's Annual of Afroasiatic Languages and Linguistics 5, 159-190.

Beckman, Jill (1997). Positional faithfulness, positional neutralization and Shona vowel harmony. Phonology 14, 1-46.

Bennett, William G. (2013). Dissimilation, consonant harmony, and surface correspondence. Ph.D. dissertation, Rutgers, The State University of New Jersey, New Brunswick, NJ.

Bennett, William G. \& Douglas Pulleyblank (). Directionality in Nkore-Kiga sibilant harmony. Linguistic Inquiry .

Boersma, Paul \& Joe Pater (2016). Convergence properties of a gradual learning algorithm for Harmonic Grammar. McCarthy, John \& Joe Pater (eds.), Harmonic Serialism and Harmonic Grammar, Equinox, Sheffield, 389-434.

Halle, Morris \& G. N Clements (1983). A problem book in phonology: a workbook for introductory courses in linguistics and in modern phonology. MIT Press, Cambridge.

Hansson, Gunnar (2001). Theoretical and Typological Issues in Consonant Harmony. Ph.D. thesis, University of California, Berkeley.

Hansson, Gunnar (2014). (Dis)agreement by (non)correspondence: inspecting the foundations. UC Berkeley Phonology Lab 2014 Annual Report: ABCConference 3-62, URL linguistics. berkeley . edu/phonlab.

Hayes, Bruce (2016). Varieties of Noisy Harmonic Grammar. Proceedings of the 2016 Annual Meeting in Phonology, USC, USC.

Hsu, Brian \& Karen Jesney (2016). Loanword adaptation in Qubec Fremch: evidence for weighted scalar constraints. Proceedings from the 34th meeting of the West Coast Conference on Formal Linguistics, Salt Lake City, UT.

Hyman, Larry M. (1998). Positional Prominence and the 'Prosodic Trough' in Yaka. Phonology 15, 41-75.

Kager, Ren (2012). Stress in windows: language typology and factorial typology. Lingua 122, 1454-1493.

Legendre, Geraldine, Antonella Sorace \& Paul Smolensky (2006). The Optimality Theory-Harmonic Grammar connection. Smolensky, Paul \& Geraldine Legendre (eds.), The Harmonic Mind, vol. 2, 339-402.

Leslau, Wolf (1967). The impersonal in Chaha. To Honor Roman Jakobson: Essays on the Occasion of his Seventieth Birthday, Mouton, The Hague, 1150-1162.

McCarthy, John (1983). Consonantal morphology in the Chaha verb. Barlow, Michael, Daniel Flickinger \& Michael Wescoat (eds.), Proceedings of the West Coast Conference on Formal Linguistics, Stanford Linguistics Association, Stanford, 176-188.

McCarthy, John (2003). OT Constraints are categorical. Phonology 20:1, 75-138.

McCarthy, John \& Alan Prince (1993). Generalized alignment. Booij, Geert \& Jaap van Marle (eds.), Yearbook of Morphology 1993, Kluwer, Dordrecht, 79-153.

McCarthy, John J. (2002). On targeted constraints and cluster simplification. Phonology 19:2, 273-292.

McPherson, Laura \& Bruce Hayes (2016). Relating application rate to morphological distance: the case of Tommo So vowel harmony. Phonology 33:1.

Potts, Christopher, Joe Pater, Karen Jesney, Rajesh Bhatt \& Michael Becker (2010). Harmonic grammar with linear programming: from linear systems to linguistic typology. Phonology 27:1, p. 77117.

Rose, Sharon (2007). Chaha (Gurage) morphology. Kaye, Alan (ed.), Morphologies of Asia and Africa, Eisenbraums, 399-424.

Shih, Stephanie \& Sharon Inkelas (2015). Morphologically conditioned tonotactics in multilevel Maximum Entropy grammar. University of British Columbia.

Shih, Stephanie S \& Sharon Inkelas (). Autosegmental Aims in Surface Optimizing Phonology. Linguistic Inquiry .

Stanton, Juliet (2016). Segmental Blocking in Dissimilation: An Argument for Co-Occurrence Constraints. Proceedings of the 2016 Annual Meeting on Phonology, USC.

Walker, Rachel (2015). Surface correspondence and discrete harmony triggers. Proceedings of the Annual Meeting on Phonology 2014.

Zoll, Cheryl (1997). Conflicting Directionality. Phonology 14.

Zymet, Jesse (2014). Distance-based decay in long-distance phonological processes. Proceedings of the 32nd West Coast Conference on Formal Linguistics, Cascadilla Press. 